\title{
EDITORIAL
}

\section{Liver toxicity: the Achilles' heel of endothelin receptor antagonist therapy?}

\author{
M.M. Hoeper
}

\section{$\mathbf{T}$} he medical community has been alerted by case reports of serious liver injury associated with sitaxentan, an endothelin receptor antagonist (ERA) used for the treatment of pulmonary arterial hypertension (PAH). The first of these case reports was published in the June issue of the European Respiratory Journal and described a patient with PAH who was initially treated with bosentan, another ERA [1]. This treatment was stopped after 6 months because of an increase in liver aminotransferases. The liver enzymes quickly returned to normal upon withdrawal of bosentan and, a few months later, sitaxentan therapy was started. Four months after this, liver aminotransferases started to increase again and the drug was discontinued, but the liver enzymes continued to rise to almost $1,000 \mathrm{U} \cdot \mathrm{L}^{-1}$ and the patient developed mild hyperbilirubinemia. Under the assumption of a drug-related idiosyncratic reaction, corticosteroid therapy was initiated resulting in prompt and sustained normalisation of liver enzymes.

In the present issue of the European Respiratory Journal, LAVELLE et al. [2] from Dublin, Ireland report on two perturbing cases of severe liver toxicity that developed 3 and 4 months, respectively, after sitaxentan therapy had been initiated. These patients showed severe liver dysfunction, with markedly elevated bilirubin levels $>300 \mu \mathrm{mol} \cdot \mathrm{L}^{-1}$ and elevated prothrombin times. In both patients, liver biopsies showed extensive hepatocellular damage and infiltrates of eosinophils and lymphocytes. As in the first case, liver function deteriorated for several weeks after discontinuation of sitaxentan. Corticosteroid therapy was not attempted. One of these patients recovered slowly, the other, however, died from pneumonia $\sim 2$ months after withdrawal of sitaxentan. At that time, bilirubin levels were still $>200 \mu \mathrm{mol} \cdot \mathrm{L}^{-1}$, indicating persistent liver dysfunction, which may have contributed to the fatal outcome.

As LAVELLE et al. [2] point out, two additional cases of hepatitis associated with sitaxentan, one of them fatal, have been observed during the long-term extension of the first clinical study with this drug in PAH patients [3]. The dose of sitaxentan in these two patients was $300 \mathrm{mg}$ b.i.d., i.e. much higher than the presently approved dosage of $100 \mathrm{mg}$ q.d.

In addition to these five cases, another, as yet unpublished, case of liver failure potentially associated with sitaxentan occurred in 2006 in Germany while the drug was still under

CORRESPONDENCE: M.M. Hoeper, Dept of Respiratory Medicine, Hanover Medical School, CarlNeuberg-Str. 1, 30625 Hanover, Germany. E-mail: hoeper.marius@mh-hannover.de study. A 40-yr-old female developed subacute liver failure after having been treated with sitaxentan at a dose of $100 \mathrm{mg}$ $q . d$. for $\sim 1.5$ yrs. Biopsy showed hepatocellular damage and areas of necrosis compatible with drug-induced liver injury. Liver function did not recover and the patient eventually underwent urgent transplantation of liver, heart and lungs at Hanover Medical School, Hanover, Germany. She is alive and well 3 yrs after the procedure. It was never ascertained whether this case was causally related to sitaxentan, as the patient was also treated with other potentially hepatotoxic drugs, such as phenprocoumon [4].

It has been known for years that ERAs, or at least bosentan and sitaxentan, that share a sulphonamide structure, can be hepatotoxic [5]. This issue is not yet clear for ambrisentan, the newest agent of this group, which chemically belongs to the propanoic acid class. So, what is the particular cause of concern in the newly reported cases? The reasons are the untypical presentations and the much more serious courses then the "usual" cases of hepatotoxicity encountered during ERA therapy. In typical cases, patients develop mildly to moderately elevated liver aminotransferases without clinical symptoms, without elevated bilirubin levels and without elevated prothrombin times. The mechanism of liver injury appears to be a direct and dose-dependent toxic drug effect. At least in the case of bosentan, an impairment of a bile salt transporter pump has been suggested to be a potential cause of liver toxicity [6]. In the vast majority of these cases, elevated liver enzymes normalise within a few weeks after dose reduction or drug discontinuation. In contrast, the patients discussed here were seriously ill with severe liver dysfunction, which was progressive for several weeks after drug discontinuation. Other potential causes of liver injury were ruled out as far as possible and liver biopsies were consistent with druginduced injury. The histological pattern, the presence of lymphocytes and eosinophils, and the clinical courses were compatible with an idiosyncratic mechanism. A similar mechanism was also suggested by the prompt response to corticosteroid therapy observed in the first patient [1].

How frequently does serious liver injury occur in patients treated with ERAs? According to information provided by the manufacturer, $>60,000$ patients have been exposed to bosentan and, as stated in the summary of product characteristics, rare cases of liver cirrhosis and liver failure have been reported. Exact numbers are not available as, in all of these cases, it was difficult to determine the precise role of bosentan due to confounding factors such as concomitant medications and 
comorbidities. More than 5,000 patients have been exposed to ambrisentan and so far there have been no reports about serious liver injury caused by this drug, but patient numbers and exposure times are not yet sufficient to conclude that ambrisentan is not hepatotoxic. The number of patients who have received sitaxentan is unknown, but can be estimated to be lower than for ambrisentan, and six cases of severe hepatitis have now been reported, four of them under the currently approved dose of $100 \mathrm{mg}$ q.d. Thus, although several lines of evidence suggest that increases in aminotransferases are less common with sitaxentan than with bosentan $[5,7,8]$, the possibility cannot be excluded that sitaxentan is afflicted with a higher potential of causing severe liver toxicity than the two other ERAs. As exact numbers are not available, and underreporting may have occurred for any of the three drugs, there is not enough evidence to decide whether this conclusion is justified. For the time being, the risk/benefit assessment remains favourable for all three ERAs. A high level of vigilance, however, is required to determine the risk of serious liver toxicity with ERAs.

Pharmacovigilance is the key. Especially in orphan diseases, drugs are often approved at a stage when there is no sufficient information about long-term safety, especially regarding rare side-effects. This is justified as the authorities have to weigh the benefits of new drugs in rare, life-threatening diseases such as PAH against the potential harm caused by drug toxicity. In the case of the three ERAs, European authorities demanded the implementation of post-marketing surveillance systems to obtain further information on the safety of these drugs after approval. These systems have gathered, and continue to gather, important safety information. The post-marketing surveillance system for bosentan was closed as planned after it had been in place for 30 months collecting data from almost 5,000 patients, but the respective databases are still open for the newer ERAs, sitaxentan and ambrisentan. The final analysis of the bosentan data was reassuring as there were no cases of fatal or permanent liver toxicity, although 10 cases were reported that fulfilled the criteria for serious druginduced liver injury [9].
For the time being, the cases reported by LAVELLE et al. [2] remind us that liver monitoring should remain an integral part of ERA therapy. Physicians are urged to report all cases of serious liver injury potentially linked to these drugs either directly to the national regulatory authorities or to any representative of the manufacturers, who are obliged to forward the reports to the authorities without delay.

\section{STATEMENT OF INTEREST}

A statement of interest for M.M. Hoeper can be found at www.erj. ersjournals.com $/ \mathrm{misc} /$ statements.dtl

\section{REFERENCES}

1 Hoeper MM, Olsson KM, Schneider A, et al. Severe hepatitis associated with sitaxentan and response to glucocorticoid therapy. Eur Respir J 2009; 33: 1518-1519.

2 Lavelle A, Sugrue R, Lawler G, et al. Sitaxentan-induced hepatic failure in two patients with pulmonary arterial hypertension. Eur Respir J 2009; 34: 770-771.

3 Barst RJ, Rich S, Widlitz A, et al. Clinical efficacy of sitaxsentan, an endothelin-A receptor antagonist, in patients with pulmonary arterial hypertension: open-label pilot study. Chest 2002; 121: 18601868 .

4 Schimanski CC, Burg J, Mohler M, et al. Phenprocoumon-induced liver disease ranges from mild acute hepatitis to (sub-) acute liver failure. J Hepatol 2004; 41: 67-74.

5 Dupuis J, Hoeper MM. Endothelin receptor antagonists in pulmonary arterial hypertension. Eur Respir J 2008; 31: 407-414.

6 Fattinger K, Funk C, Pantze M, et al. The endothelin antagonist bosentan inhibits the canalicular bile salt export pump: a potential mechanism for hepatic adverse reactions. Clin Pharmacol Ther 2001; 69: 223-231.

7 Barst RJ, Langleben D, Badesch D, et al. Treatment of pulmonary arterial hypertension with the selective endothelin-A receptor antagonist sitaxsentan. J Am Coll Cardiol 2006; 47: 2049-2056.

8 Benza RL, Mehta S, Keogh A, et al. Sitaxsentan treatment for patients with pulmonary arterial hypertension discontinuing bosentan. J Heart Lung Transplant 2007; 26: 63-69.

9 Humbert M, Segal ES, Kiely DG, et al. Results of European postmarketing surveillance of bosentan in pulmonary hypertension. Eur Respir J 2007; 30: 338-344. 